\title{
A New Method for Edge Extraction in Images using Local Form Factors
}

\author{
Supratim Gupta \\ Department of Electrical \\ Engineering, \\ Indian Institute of Technology, \\ Kharagpur, India
}

\author{
Aurobinda Routray \\ Department of Electrical \\ Engineering, \\ Indian Institute of Technology, \\ Kharagpur, India
}

\author{
Anirban Mukherjee \\ Department of Electrical \\ Engineering, \\ Indian Institute of Technology, \\ Kharagpur, India
}

\begin{abstract}
This article proposes a new spatial domain measure of local energy to extract the image features like edges. We define the measure as the local form factor (FF). It is the ratio of RMS to average of the pixel values in a region. Inverse square of the local FF around a center pixel is defined as an index of edge strength at that pixel. The proposed method could be applied directly on any image without smoothing for noise removal. It only needs an estimate of the Signal-to-Noise ratio (SNR) of the images to compensate the effect of noise. The compensated feature image is passed through non-minimum suppression and universal thresholding processes to produce the final edge map. The performance of the method is assessed using Baddeley Error Metric (BEM) and compared with those resulted from the popular Canny edge detector with different scales. The experimental results are encouraging the application of the method to extract edges and hence can be used as a potential candidate for general feature extraction.
\end{abstract}

\section{General Terms}

Image feature extraction, Low-level image processing

\section{Keywords}

Baddeley Error Metric, Canny edge detector, Edge detection, Local form factor, Non-minimum suppression.

\section{INTRODUCTION}

Extraction of Image features like edges, lines etc. finds its application in image analysis as well as computer vision for several decades. It requires a number of local image preprocessing techniques [1-4]. A considerable research has been performed for the development of optimal edge detectors using various models.

A gradient based detector has used masks to approximate the first derivative of intensity at an image pixel to find the edges [1-2]. Performance of these convolution based methods depends on the size of the region bounded by the edges and achieves accuracy only for specific images [1]. On the other hand, Laplacian operator finds the second derivative to determine the edge magnitude at each pixel in an image using mask of different sizes. It may produce double response to a few types of edges [1-2], [5]. The later development in this line such as [4], [6-8] utilizes the fact that second derivative crosses zero value at the edge pixel in an image. These methods are highly sensitive to noise and use a smoothing filter to remove it at the cost of higher inaccuracy in the edge location. Literature reports that the Gaussian smoothing may lead to suboptimal detection of edges [1]. Thus choice of the smoothing scale is crucial. However no systematic method exists to find a suitable scale for an optimum performance. Moreover, all these methods are highly sensitive to illumination variations. Recent work on edge detection by Scotney et al. [9] employs a near circular operator. Here the Gaussian smoothing is embedded in the operator and thus performance depends on choice of scale.

In [6] Canny has developed an optimal detector for step edges. It can detect other types of edges such as roofs, lines and gratings etc. with limited accuracy. Amongst all the Canny's method has been the most popular choice as reported in the literature [4], [6].

It is observed that a significant variation in local energy indicates the existence of edges in that region [10-11]. It suggests that the congruency of phase achieves maximum at the edge pixel. This method essentially operates on image in the frequency domain. Here, the choice of filter kernel may affect the output of the detector. The performance is also dependent on the amount of frequency spread and scales considered for the measurement [12-14]. The weights to the local energy that alleviates the problem with low frequency spread are computed using arbitrarily fixed weighting fraction and a gain. It is also reported that being a normalized dimensionless index the PC is highly sensitive to noise [12-14]. The effect of noise that is considered to be uniform in all orientations is removed by a choice of threshold that is also dependent on a set of constants determined by trial and error. Finally, the computational complexity is high for this method [12]. The high computational burden is due to large number of convolution operations with filters for many wavelet scales and orientations.

Other technique such as SUSAN is also related to gradient measure and thus may be sensitive to noise [15]. Here, the choice of user settable thresholds affects the detection performance. An improved SUSAN technique has been reported in [16]. However, the complexity of this algorithm increases two fold as compared to the original SUSAN proposed in [15].

The multi-scale edge detection reported in the literature may not exhibit good performance as the feature count and the location varies at different scales [11-14]. Albeit, the computational burden of these algorithms is often high for practical uses [17-18].

An "on and off" edge filter based on learning probability distribution has been reported in [19-20]. Here, the learning is based on gradient edge map. The performance has been tested using misclassification error of edge pixel. However, the misclassification error has been reported to be a poor performance indicator as far as edge detection is concerned [21]. 
Moreover, choice of filter scale during learning and test may influence the outcome.

The aim of this paper is to develop an approach of edge detection based on the measurement of energy in local region in an image. To meet the goal we propose a novel measure of local energy. We call it the form factor (FF). Inverse of square of FF in a region gives the edge strength. As will be evident, the proposed method could be applied directly on any image without any pre-smoothing. The FF is computed within a $3 \times 3$ region around a centre pixel. This method only needs an estimate of the Signal-to-Noise ratio (SNR) of the images to compensate for the effect of noise. The SNR is estimated using the method proposed by Sim et al. [22]. The compensated feature image is passed through non-minimum suppression (as local energy is inversely proportional to edge strength here) within $5 \times 5$ window and a universal thresholding processes to produce the final edge map.

The qualitative and quantitative results are compared with those resulted from the Canny's detector with different scales. These results confirm the suitability of the method to extract edges and hence can be used as a potential candidate for general feature extraction.

Section-II describes the proposed method of edge detection. In Section-III the experimental results on images with various types of edges along with discussion are presented. The conclusion and the future scope of the work are presented in section IV.

\section{THE PROPOSED METHOD}

\subsection{Local Form Factor (FF) in Image}

We define local form factor in an image as the ratio of r.m.s to average of pixels sequence ( $\mathbf{x} \in \mathfrak{R}_{P}^{N}$ ) within a region as:

$$
F=\frac{R \cdot M \cdot S}{\text { Average }}=\sqrt{\left(1+\frac{\sigma^{2}}{\mu^{2}}\right)}=\sqrt{N} \frac{\|\mathbf{x}\|_{2}}{\|\mathbf{x}\|_{1}}
$$

where, $\mu$ and $\sigma$ are mean and standard deviation of pixel values in the region respectively, $\|\cdot\|_{p}$ is the $p$ norm of the pixel sequence, and $N$ is the number of pixels.

\subsection{The Edge Strength from FF}

The form factor defined in (1) is not a normalized quantity. In this section we define inverse of square of FF as a normalized index to quantify the image feature like an edge. The normalization facilitates the adoption of universal thresholds for regions of different sizes. This index indicates the edge strength (ESI) at the centre pixel within a small region. Thus the ESI can be expressed as:

$$
\alpha=\frac{1}{F^{2}}=\frac{\|\mathbf{x}\|_{1}^{2}}{N\|\mathbf{x}\|_{2}^{2}}
$$

In any image two broad types of region like homogeneous, and non- homogeneous that contains edges, lines, corners etc. may exist. In homogeneous regions the variance is zero and hence the ESI is unity. The non uniform regions will result in ESI values less than unity. Thus the maximum value of ESI is 1 . The minimum limit is reached when the ratio of variance to mean becomes largest. This is possible when the variance is the highest and mean is the lowest. A vector (element values within 0 to 255) of all but one non-zero element satisfies the condition for the ratio to be minimum. Thus the range of ESI is as follow

$$
\frac{1}{N} \leq \alpha \leq 1
$$

All $\alpha$ values within its range do not represent an edge pixel. A coarse idea on the values of ESI for edge can be obtained from the relationship between $\alpha$ and entropy of a region. We find that ESI varies inversely with entropy. It can be expressed as in (4) for a Gaussian distribution [23]

$$
\alpha=\frac{\mu^{2}}{\mu^{2}+\frac{1}{2 \pi} e^{(2 H-1)}}
$$

where $H$ is the entropy of the region. The relation in (4) holds as entropy is logarithmically proportional to variance of signal concerned [23]. It follows that the extreme values of entropy produce extreme values of ESI. It is evident that a region with low entropy is completely uniform while the high entropy is associated with higher degree of irregularities in the region. Thus prominent edges cannot exist in the region with such extreme values of entropy or ESI. Therefore the ESI values of an edge pixel will tend to lie mid way within its range. Statistical test results indicate the boundary of ESI for an edge as:

$$
0.5 \leq \alpha^{e d g e} \leq \alpha_{\text {high }}
$$

where, $\alpha_{\text {high }}$ is close to unity and limits the contrast level up to which edges (weak edges) are to be detected. The range of ESI in (5) implies that an edge exists in a region where RMS value of pixels is less than $\sqrt{2}$ times of their average value. Fig. 1 depicts the edge strength values for a step edge in 1-D (similar to different level of contrast in image). Here, $\alpha$ is computed within a moving window of constant size. It is apparent from the figure that the ESI falls within the range as in (5) in presence of an edge.

\subsection{ESI under Zero-mean Gaussian Noise}

This section analyses the application of ESI in images corrupted with zero-mean random Gaussian noise. From (1) and (2) we can express the ESI of a noisy image as

$$
\alpha_{g}=\frac{\mu_{g}^{2}}{\mu_{g}^{2}+\sigma_{g}^{2}}
$$

where $\mu_{g}$ and $\sigma_{g}^{2}$ are the overall mean and variance of the region with noise. Replacing the terms in (7) by the means $\left(\mu_{s}\right.$ and $\left.\mu_{n}=0\right)$, the variances $\left(\sigma_{s}^{2}, \sigma_{n}^{2}\right)$ and the covariance $\left(\sigma_{n s}^{2}\right.$ 
) of the noise free image region and noise the relation between ESI of noise free image $\alpha_{s}$ and $\alpha_{g}$ can be found as

$$
\frac{1}{\alpha_{g}}=\frac{1}{\alpha_{s}}+\frac{1}{\alpha_{n}}
$$

where $\alpha_{n}=\frac{\mu_{s}^{2}}{\sigma_{n}^{2}+2 \sigma_{n s}^{2}}$ is due to noise. Here the term $\sigma_{n s}^{2}$ (covariance) can be neglected as the noise is uncorrelated with the image.

Thus $\quad \alpha_{n}=\frac{\mu_{s}^{2}}{\sigma_{n}^{2}}=\frac{S N R}{F_{s}^{2}-1}$

where $F_{s}^{2}=\frac{1}{\alpha_{s}}$ and $S N R=\frac{\sigma_{s}^{2}}{\sigma_{n}^{2}}$ is the Signal-to-Noise-Ratio

(SNR) measured in linear scale. So from (2), (8) and (9) we get

$$
\alpha_{s}=\frac{1+\frac{1}{S N R}}{\frac{1}{\alpha_{g}}+\frac{1}{S N R}}
$$

From (10) it is inferred that the feature value of noise free image can directly be computed from the form factor of noisy image and the SNR.

It is observed that the noise term in (9) lowers the minimum value of ESI. Thus it also affects the lower limit of the ESI for an edge and the change is dependent on the SNR value. However, noise compensation prevents this change and keeps the ESI range fixed.

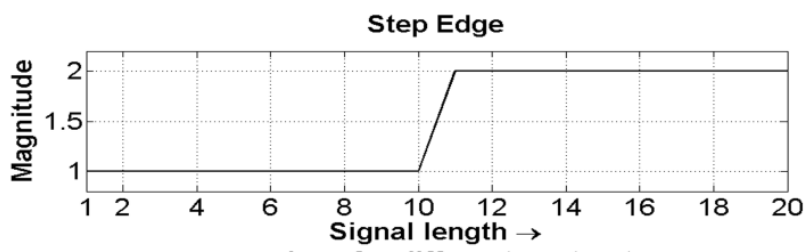

$\alpha$ values for different contrast

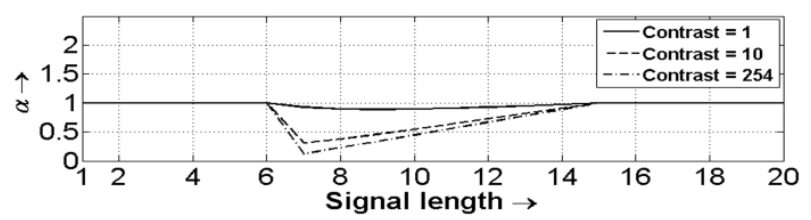

Fig. 1: Range of $\alpha$ of edge points for step edge

\subsection{Estimation of SNR from Single Image}

For removal of the effect of noise on the ESI as observed in (10) an estimation of SNR is desirable. An autocorrelation based method can be applied for the purpose. In the present work we obtain the estimate of the SNR for a region following the method developed in [22]. It includes computation of mean and autocorrelation peak at origin of the image region which is contaminated by zero-mean Gaussian noise. The autocorrelation peak of the image without noise is estimated by first order neighborhood extrapolation method from two autocorrelation values nearest to its peak.

\subsection{Algorithm for Proposed Methodology}

The steps of the algorithm are presented in the flow chart as in Fig. 2.The $\alpha_{\text {high }}$ is decided by the saliency of the weak edges to be detected. The edge index for noise free image is computed following (10) within a moving window of size $3 \times 3$.

The algorithm generates a noise compensated ESI-map of the image. The ESI values are supposed to attain minimum in the direction of lowest variance along the true edge. However, there may be spreading around it due to local moving windowed operations. To remove this ESI map is passed through a NonMinimum Suppression (NMS) algorithm along the direction of lowest variance within the window (of size $=5 \times 5$ ) around each pixel. This retains the lowest of the ESI values generated from the multiple responses around the true edges. Finally edgethresholding as in (5) is applied to generate the edge map.

\section{EXPERIMENTS AND DISCUSSION}

In this section we have presented both the qualitative and the quantitative performance of the proposed algorithm. A number of standard images referred in different literature are considered for qualitative assessment [13-14], [15-18]. The images contaminated with different SNRs are subjected to the proposed algorithm. In the second phase of experiment we consider quantitative performance of our algorithm on randomly selected sixty images from Berkley Segmentation Dataset (BSDS) [24]. The Baddeley Error Metric (BEM) has been chosen as the quantitative performance measure. The relative performance has been evaluated with respect to Canny's method as it has been accepted as some short of standard by several authors.

\subsection{Edge Map of Images without Noise}

In this subsection we have presented the results of our proposed method and those by Canny's edge detector for the noise free images containing different type of edges and regions of varying contrast.

In Fig. 3 we have considered a simple image that contains a small embedded. In this figure the intensity level of the square and surrounding regions are 0 and 1 . This is considered to demonstrate the effectiveness of the proposed algorithm for a contrast level as low as 1 for gray scale images which are invisible to human eye too. The output from Canny's method with default scale 1 is also presented for comparison. A little distortion can be seen in this edge map. The detection of weak edges is limited by the choice of the upper bound of edge strength index (ESI). It can be associated with local entropy which is inversely proportional to ESI. It is apparent that the entropy of an edge region will assume the mid values in the range. Similarly the ESI for edges also falls in the mid-way of its range. The image features such as edges assume a value within $\left[0.5, \alpha_{\text {high }}\right]$. The upper limit of the feature value determines the contrast level across weak edge that can be detected by the method. Empirically, we can find out that $\alpha_{\text {high }}$ may be varied in the range of $0.85,0.9996$. 
It can be observed that the above image contain only the step edges. There are many other types of edges such as gratings and curved edges etc. [13].

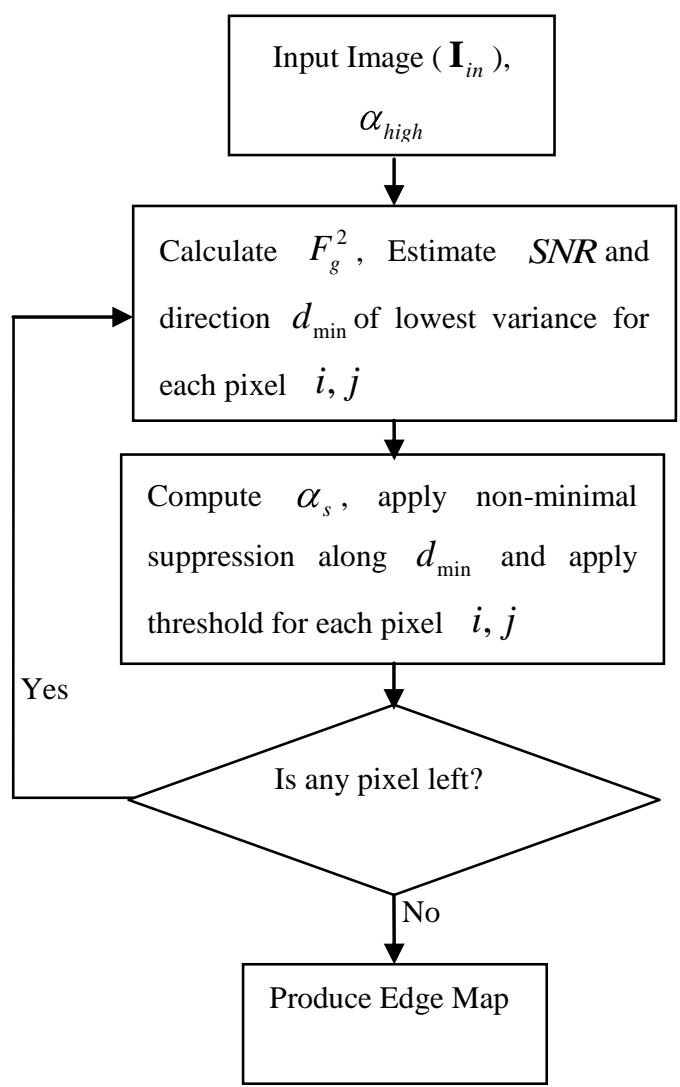

Fig. 2: Flow chart of the algorithm

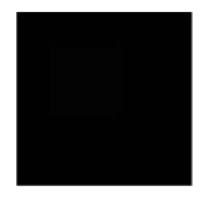

(a)

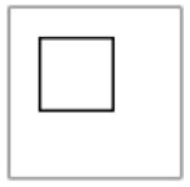

(b)

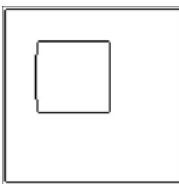

(c)
Fig. 3: (a). Original image with low contrast and lower pixel value, (b). Edge image by proposed method, (c) Edge image by Canny edge detector

We have considered images as in Fig. 4(a) and Fig. 5(a) that contain such types of edges. Gratings in image (Fig. 4) may exist in practice due to various orientations of sources of light and those of imaging devices. On the other hand in Fig. 5a a star like curved edge is present in a varying contrast region. We have shown the results obtained from Canny's method in Fig. 4(b)-(d), Fig. 5(b)-(d) for different scales of the smoothing filter. In Fig. 4(e), Fig. 5(e), the edge maps by the proposed method without the non-minimum suppression (NMS) are presented. These figures show that our algorithm is capable of capturing all the required edges. However, widths of these edges are not of single pixel. Therefore, we apply NMS within $3 \times 3$ as well as $5 \times 5$ moving region to obtain edge-maps as presented in Fig. 4(f)-(g), Fig. 5(f)-(g).

In Fig. 4 it can be observed that Canny's method produces multiple edges around the true edge even after non-maximum suppression. This is because the edges in the original image change their edginess from step to line for which the Canny detector may not be effective [13-14] even after extensive experiments to find possible improvements with change of scales. It is found that our algorithm finds a single edge for the same image as it only measures the local energy variation instead of the gradient measure. It should also be noted that the edges in such cases as well as for the other images may not be localized accurately as the smoothing scale is increased. On the other hand spurious edges are observed when the scale is reduced.

\subsection{Edge Map of Images with Noise}

Under practical situations, the performance of any edge detection algorithm is needed to be judged in presence of noise. We have added zero-mean Gaussian noise of different Signal-to-Noise Ratio (SNR) to the image as shown Fig. 5(a). Such a noisy image with $15 \mathrm{~dB}$ SNR is shown in Fig. 6(a). The results of the Canny's as well as the proposed algorithm are shown in Fig. 6(b) to Fig. 6(h).

In this case it can be noticed that Canny's method produces higher false negative for all scale as well as higher localization error for the higher scale. The better performance of our algorithm can be attributed to the integral nature of the proposed edge index. However, the index is not fully insensitive to such noises. So a noise compensation of the index is performed. The result is shown in Fig. 6(f)-(h). Since our method does not require any pre-smoother better localization of edge may be observed in the figures shown.

Finally, from Fig. 5 - Fig. 6 we observe that the best results are obtained by Canny's method with the scale of 1 and 0.75 respectively. Thus scale of the smoother influence the output of the detector. We found consistently good results for our algorithm on the same images and noticed that the proposed method with $5 \times 5$ NMS produces better results than that of $3 \times 3$ NMS.

\subsection{Quantitative Evaluation}

\subsubsection{The Dataset}

An extensive analysis of the proposed algorithm has been performed on randomly selected sixty images from the Berkley Segmentation Dataset (BSDS) [24]. It consists of a wide range of natural images along with 5-9 binary edge maps for each of these images obtained by human subjects. These binary edge images can be considered as ground truth for comparison of the output of any edge detection algorithm [24-26]. The images in this dataset are of sizes $321 \times 481$ and $481 \times 321$.

\subsubsection{The Perfomance Index}

Literature suggests a) good detection, b) good localization, and c) single response to an edge as three criteria [1], [6], [13-14], [21], [27-28] on the performance of edge detectors. Canny had shown that the first two criteria contradict each other [6], [21]. The third criterion is generally alleviated by employing 
non-maximum suppression followed by a hysteresis thresholding. In the present algorithm we have used non minimum suppression as the ESI is inversely related to local energy. Therefore a performance index for edge detector is primarily concerned with the first two criteria.

Studies suggest few indices generally employed to assess the performance of an edge detector with the available ground truth [18], [21], [25], [29-30]. These are as Misclassification Error, Pratt's Figure of Merit (FOM), and Baddeley Error Metric (BEM). The misclassification error is related to finding the probability of incorrect classification for every pixel in an image. It is evident that this index may be incapable of capturing inaccuracies due to shape distortion of object boundaries. This leads to the edge localization error. A detail review on this index can be found in [30]. The Pratt's FOM suffers from the above anomaly too. In addition to this the index is found to be insensitive to false negative error. The proper theoretical justification is also unavailable [21]. The BEM proposed in [21] has been considered in several research works for measuring errors in detection and localization. This index is devoid of the shortcomings prevailed in the earlier two [25], [31].

The BEM is computed using Hausdorff distance for binary images [21]. The metric is expressed as follow -

$$
\Delta_{w}^{p} \quad I, I_{r e f}=\left[\frac{1}{N} \sum_{x \in X}\left|w(d(x, I))-w\left(d\left(x, I_{r e f}\right)\right)\right|^{p}\right]^{\frac{1}{p}}
$$

Where $d(x, I)$ is the shortest distance from $x \in X$ to $I \subseteq X, w(t)$ is a continuous function on $0, \infty$, concave and strictly increasing and $1 \leq p \leq \infty$. The transformation $w(t)$ is defined in [21] as $w(t)=\min (t, c)$ for a fixed $c>0$.

The lower value of $\Delta_{w}^{p}$ indicates that $I$ is close to $I_{r e f}$ [21], [2526], [31]. In the present article we choose to use $\Delta_{w}^{2}$ as the performance index for the proposed edge detector.
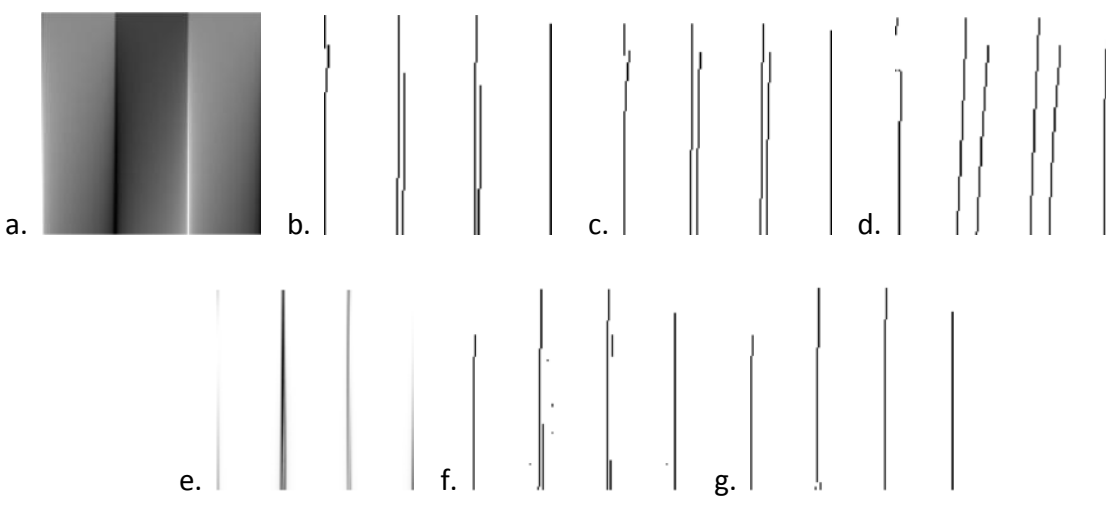

Fig. 4: (a). Original image with gratings, (b). Canny edge detection with $\sigma=0.75$, (c) Canny edge detection with $\sigma=1$, (d). Canny edge detection with $\sigma=3$, (e). Proposed edge detection without NMS, (f) Proposed edge detection with $3 \times 3$ NMS, (g) Proposed edge detection with $5 \times 5 \mathrm{NMS}$
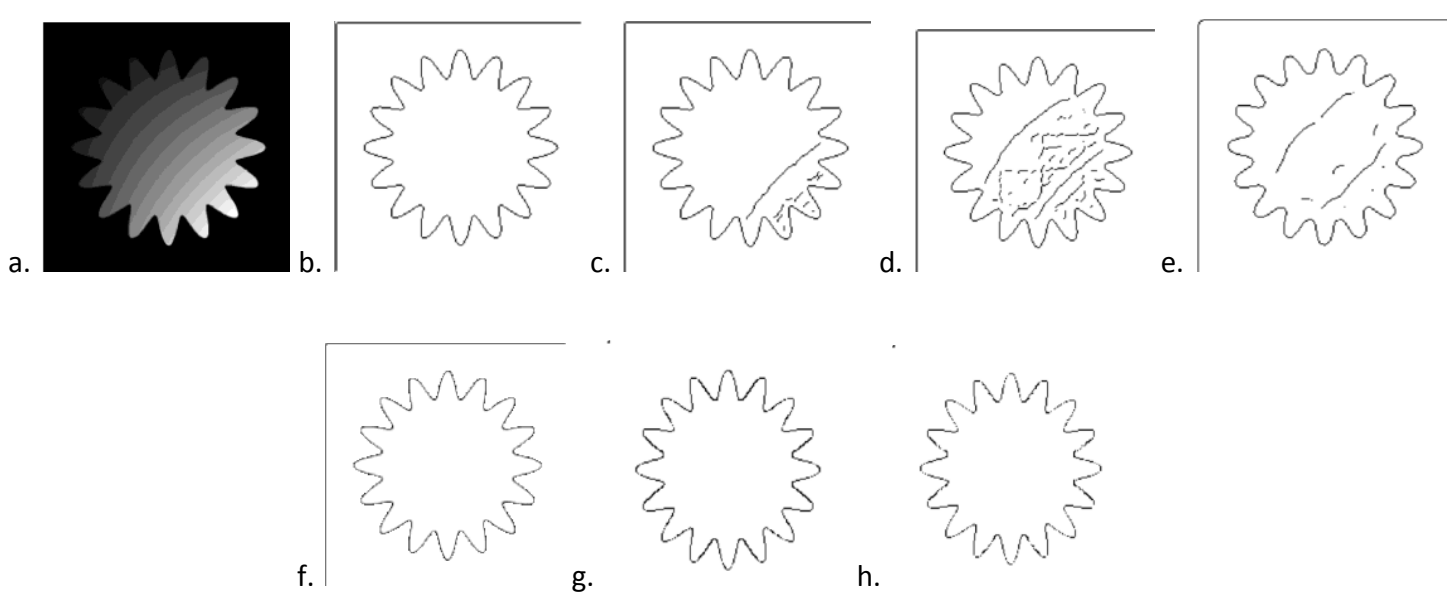

h.

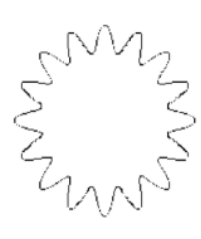

Fig. 5: (a). Original image without noise, (b). Canny edge detection with $\sigma=0.75$, (c) Canny edge detection with $\sigma=1$, (d). Canny edge detection with $\sigma=1.5$, (e). Canny edge detection with $\sigma=3$, (f). Proposed edge detection without NMS, (g) Proposed edge detection with $3 \times 3$ NMS, (h) Proposed edge detection with $5 \times 5$ NMS 

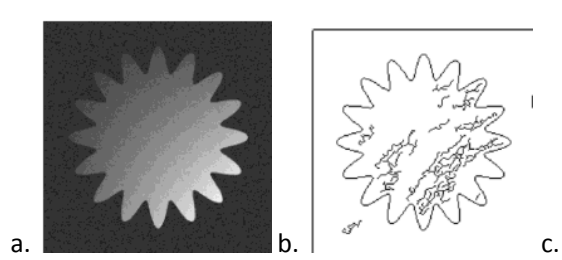

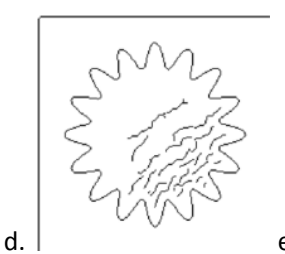

e.

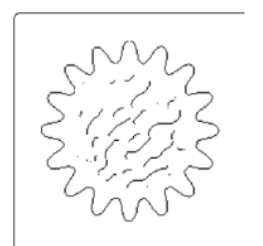

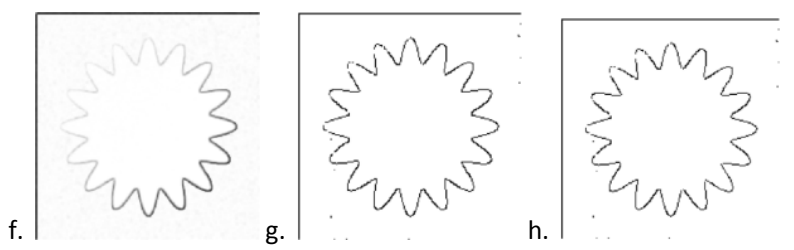

Fig. 6: (a). Original image with noise of 15dB SNR, (b). Canny edge detection with $\sigma=0.75$, (c) Canny edge detection with $\sigma=1$, (d). Canny edge detection with $\sigma=1.5$, (e). Canny edge detection with $\sigma=3$, (f). Proposed edge detection without NMS, (g) Proposed edge detection with $3 \times 3$ NMS, (h) Proposed edge detection with $5 \times 5$ NMS

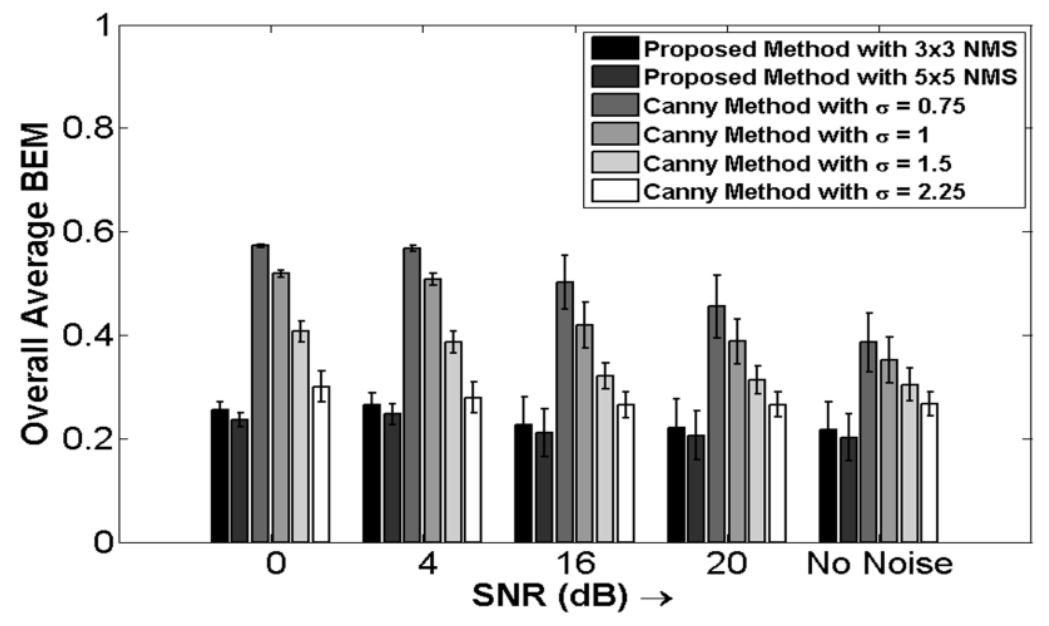

Fig. 7: Overall average BEM on BSDS image dataset different noise condition for proposed method with $3 \times 3$ NMS, $5 \times 5$ NMS, Canny edge detector with different scale $(\sigma)$.

\subsubsection{The Experiment}

The experimental procedure for quantitative evaluation of the edge detector on a given image $I$ taken from the BSDS can be performed by the following algorithm-

1) Compute binary edge map for a given image with and without noise following the algorithm depicted in Fig. 2. Here we choose weak edge detection limit as $\alpha_{\text {high }} \in[0.8,1)$ as 0.90 .

2) Compute $\Delta_{w}^{2}$ by considering each of the human-made segmented image as reference and the generated edge map as test image using (11). Here the scale factor $C$ is equal to $\sqrt{321^{2}+481^{2}} \approx 578$. In this case the BEM in (11) lies within 0,1 .

3) Average all the BEM to produce a single measure of performance of an edge detector for an image.
Same performance indices are computed similarly on the same dataset for the Canny's edge detector. The average BEM over the whole dataset for the images without noise and those for noise level of SNR $=0,4,16,20 \mathrm{~dB}$ are shown in Fig. 7 for Canny's method with different scales and the proposed algorithm with $3 \times 3$ and $5 \times 5$ NMS.

We find a consistent low BEM for the proposed algorithm as compared to the Canny edge detector. We have also investigated with increasing scale $\left(\sigma^{2}>3\right)$ for Canny's edge detector. However, it produces higher BEM. The results obtained for the Canny edge detector may be attributed by the uncertainty between the detection and localization of edges [6], [21]. On the other hand, the proposed algorithm does not use any pre-filter, instead a noise compensation is made in the feature image during post processing. Thus it avoids the localization error.

In the proposed algorithm the upper bound of ESI is set to 0.9. It can be noted that changes in the upper bound of the index may influence the results as it will either introduce or omit a few weak edges. The decision on the upper bound of the feature 
index can be made based on the context i.e. how far we need to detect and localized the weak edges.

Finally, we found form these measurements that our algorithm with $5 \times 5$ NMS produces best results on an average throughout the image data set.

\section{CONCLUSION}

A new and simple local energy measure called the form factor (FF) is introduced. Based on this measure a novel edge detection algorithm has been proposed. The edge strength computed as inverse of the square of FF. The novelty of this index can be summarized as below -

- It is a dimensionless and normalized index.

- The effect of noise can easily be alleviated using an estimation of SNR for zero-mean Gaussian noise.

- It does not require any pre-smoothing. Thus the uncertainty between edge detection and localization is avoided and better accuracy can be achieved.

The experimental validation proves its effectiveness for detection of edges in natural images under different noise conditions. In the current article a zero-mean Gaussian noise model has been considered. However, noise compensation for other noise models (such as multiplicative or speckle noise) can be explored further.

\section{REFERENCES}

[1] Sonka M., Hlavac V., and Boyle R., "Image Processing, Analysis, and Machine Vision," $2^{\text {nd }}$ Ed., Singapore: Thomson Learning Asia Pte. Ltd., 1998.

[2] Gonzalez R. C., and Woods R. E., "Digital Image Processing," $3^{\text {rd }}$ Ed., vol. 2. Singapore: Pearson Education Singapore Pte. Ltd., 2008.

[3] Pellegrino F. A., Vanzella W., and Torre V., "Edge Detection Revisited," IEEE Trans. Syst., Man, and Cybern._Part B: Cybern., vol. 34, no. 3, pp. 1500-1518, Jun., 2004.

[4] Rakesh R. R., Chaudhuri P., and Murthy C. A., "Threshonding in Edge Detection: A Statistical Approach," IEEE Trans. Image Process., vol. 13, no. 7, pp. 927-936, Jul., 2004.

[5] Jain A. K., "Fundamentals of Digital Image Processing," $4^{\text {th }}$ Indian reprint, Singapore, Pearson Education Pte. Ltd., 2005.

[6] Canny J., "A Computational Approach to Edge Detection," IEEE Trans. Pattern Anal. and Mach. Intell., vol. PAMI-8, no. 6, pp. 679-698, Nov., 1987.

[7] Sarkar S., and Boyer K. L., "Optimal Infinite Impulse Response Zero Crossing Based Edge Detectors," CVGIP: Image Understanding, vol. 54, no. 2, pp. 224-243, Sep., 1991.

[8] Marr D., and Hildreth E., "Theory of Edge Detection," in Proc. Roy. Soc. of London, Series B, Biological Sci., vol. 207, no. 1167, Feb., 1980, pp. 187-217.

[9] Scotney B. W., and Coleman S. A., "Improving angular error via systematically designed near-circular Gaussian- based feature extraction operators," Pattern Recognition, vol. 40, no. 5, pp. 1451-1465, May, 2007.

[10] Morrone M. C., and Burr D. C., "Feature Detection in Human Vision: A Phase-Dependent Energy Model," in Proc. Roy. Soc. of London, Series B, Biological Sci., vol. 235, no. 1280, Dec., 1988, pp. 221-245.

[11] Morrone M. C., and Owens R. A., "Feature Detection from Local Energy," Pattern Recognition Letts., vol. 6, no. 5, pp. 303-313, Dec., 1987.

[12] Kovesi P., "Invariant Measures of Image Features from Phase Information," Ph.D. Desertation, Dept. Psychology, Western Australia Univ., May, 1996.

[13] Kovesi P., " Phase Congruency Detects Corners and Edges," in Proc. $7^{\text {th }}$ Int. Conf. Digital Image Computing: Techniques and Applicat., Sydney, Australia, Dec., 2003, pp. 309-318.

[14] Kovesi P., "Edges Are Not Just Steps," in Proc. $5^{\text {th }}$ Asian Conf. on Comput. Vision, Melbourne, Australia, Jan., 2002, pp. 822-827.

[15] Smith S. M., and Brady J. M., "SUSAN-A New Approach to Low Level Image Processing," Int. J. Comput. Vision, vol. 23, no. 1, pp. 45-78, 1997.

[16] Bankman I. N., and Rogala E. W., "Corner Detection for Identification of Man-Made Objects in Noisy Aerial Images," in Proc. Int. Conf. Soc. of Photographic Instrumentation Engineers, vol. 4726, 2002, pp. 304-309.

[17] Coleman S. A., Scotney B. W., and Suganthan S., "Multiscale edge detection on range and intensity images," Pattern Recognition, vol. 44, no. 4, pp. 821-838, Apr., 2011.

[18] Yi S., Labate D., Easley G. R., and Krim H., "A Shearlet Approach to Edge Analysis and Detection," IEEE Trans. Image Process., vol. 18, no. 5, pp. 929-941, May, 2009.

[19] Konishi S., Yuille A. L., Coughlan J. M., and Zhu S. C., "Statistical Edge Detection: Learning and Evaluating Edge Cues," IEEE Trans. Pattern Anal. and Mach. Intell., vol. 25, no. 1, pp. 57-74, Jan., 2003.

[20] Konishi S., Coughlan J. M., and Yuille A. L., "Statistical Approach to Multi-Scale Edge Detection," Image Vision and Comput., vol. 21, no. 1, pp. 37-48, Jan., 2003.

[21] J. A. Baddeley, "An error metric for binary images," in Proc. IEEE Workshop on Robust Comput. Vision, Bonn, 1992, pp. 59-78.

[22] Sim K. S., Lai M. A., Tso C. P., and Teo C. C., "Single Image Signal-to-Noise Ratio Estimation for Magnetic Resonance Images,” J. Med. Syst., Springer Link, Jul., 2009, doi 10.1007/s10916-009-9339-9.

[23] Mukherjee D., and Ratnaparkhi M. V., "On the Functional Relationship between Entropy and Variance with Related Application," Commun. in Stat. - Theory and Methods, vol. 15, no. 1, pp. 291-311, 1986.

[24] Martin D., Fowlkes C., Tal D., and Malik J., "A Database of Human Segmented Natural Images and its Application to Evaluating Segmentation Algorithms and Measuring 
Ecological Statistics," in Proc. $8^{\text {th }}$ Int. Conf. Comput. Vision, vol. 2, Jul., 2001 , pp. 416-423.

[25] Lopez-Molina C., Bustince H., Fernandez J., Couto P., and Baets B. D., "A Gravitational Approach to Edge Detection based on Triangular Norms," Pattern Recognition, Elsivier, vol. 43, no. 11, pp. 3730-3741, Nov. 2010.

[26] Lopez-Molina C., Bustince H., Fernandez J., Barrenechea E., Couto P., and Baets B. D., "A t-Norm Based Approach to Edge Detection," Bio-Inspired Systems: Computational and Ambient Intell., Lecture notes in Comput. Sci., vol. 5517, pp. 302-309, 2009.

[27] Venkatesh S., and Kitchen L. J., "Edge Evaluation Using Necessary Components," Comput. Vision, Graph. and Image Process., vol. 54, no. 1, pp. 23-30, Jan., 1992.

[28] Demigny D., Lorca F. G., and Kessal L., "Evaluation of edge detectors performances with a discrete expression of
Canny's criteria," in Proc. Int. Conf. Image Process., vol. 2, Oct., 1995, pp. 169-172.

[29] Pratt W. K., "Digital Image Processing," $3^{\text {rd }}$ Ed., John Wiley \& Sons inc., Singapore, 2003.

[30] Bowyer K., Kranenburg C., and Dougherty S., "Edge Detector Evaluation Using Empirical ROC Curves," Comput. Vision and Image Understanding, Elsevier, vol. 84, no. 1, pp. 77-103, Oct., 2001.

[31] Medina-Carnicer R., Carmona-Poyato A., Munoz-Salinas R., and Madrid-Cuevas F.J., "Determining Hysteresis Thresholds for Edge Detection by Combining the Advantages and Disadvantages of Thresholding Methods," IEEE Trans. Image Process., vol. 19, no. 1, pp. 165-173, Jan., 2010 